\title{
On Interpretability Between Some Weak Essentially Undecidable Theories
}

\author{
Lars Kristiansen $^{1,2(\bowtie)}$ and Juvenal Murwanashyaka ${ }^{1}$ \\ 1 Department of Mathematics, University of Oslo, Oslo, Norway \\ \{larsk, juvenalm\}@math . uio.no \\ 2 Department of Informatics, University of Oslo, Oslo, Norway
}

\begin{abstract}
We introduce two essentially undecidable first-order theories WT and T. The intended model for the theories is a term model. We prove that WT is mutually interpretable with Robinson's R. Moreover, we prove that Robinson's Q is interpretable in T.
\end{abstract}

\section{Introduction}

A first-order theory $T$ is undecidable if there is no algorithm for deciding if $T \vdash \phi$. If every consistent extension of an undecidable theory $T$ also is undecidable, then $T$ is essentially undecidable.

We introduce two first-order theories, WT and T, over the language $\mathcal{L}_{\mathrm{T}}=$ $\{\perp,\langle\cdot, \cdot\rangle, \sqsubseteq\}$ where $\perp$ is a constant symbol, $\langle\cdot, \cdot\rangle$ is a binary function symbol and $\sqsubseteq$ is a binary relation symbol. The intended model for these theories is a term model: The universe is the set of all variable-free $\mathcal{L}_{\mathrm{T}}$-terms. Each term is interpreted as itself, and $\sqsubseteq$ is interpreted as the subterm relation $(s$ is a subterm of $t$ iff $s=t$ or $t=\left\langle t_{1}, t_{2}\right\rangle$ and $s$ is a subterm of $t_{1}$ or $\left.t_{2}\right)$.

The non-logical axioms of WT are given by the two axiom schemes:

$$
s \neq t
$$

where $s$ and $t$ are distinct variable-free terms.

$$
\forall x\left[x \sqsubseteq t \leftrightarrow \bigvee_{s \in \mathcal{S}(t)} x=s\right]
$$

where $t$ is a variable-free term and $\mathcal{S}(t)$ is the set of all subterms of $t$. There are no other non-logical axioms except those given by these two simple schemes, and at a first glance WT seems to be a very weak theory. Still it turns out that Robinson's essentially undecidable theory R is interpretable in WT, and thus it follows that also WT is essentially undecidable. The theory $\mathrm{T}$ is given by the four axioms:

$$
\begin{aligned}
& \mathrm{T}_{1} \forall x y[\langle x, y\rangle \neq \perp] \\
& \mathrm{T}_{2} \forall x_{1} x_{2} y_{1} y_{2}\left[\left\langle x_{1}, x_{2}\right\rangle=\left\langle y_{1}, y_{2}\right\rangle \rightarrow\left(x_{1}=y_{1} \wedge x_{2}=y_{2}\right)\right] \\
& \mathrm{T}_{3} \forall x[x \sqsubseteq \perp \leftrightarrow x=\perp] \\
& \mathrm{T}_{4} \forall x y z[x \sqsubseteq\langle y, z\rangle \leftrightarrow(x=\langle y, z\rangle \vee x \sqsubseteq y \vee x \sqsubseteq z)] .
\end{aligned}
$$


It is not difficult to see that $\mathrm{T}$ is a consistent extension of WT. Thus, since WT is essentially undecidable, we can conclude right away that also $\mathrm{T}$ is essentially undecidable. Furthermore, since every model of the finitely axiomatizable theory $\mathrm{T}$ is infinite, $\mathrm{T}$ cannot be interpretable in WT, and the obvious conjecture would be that $\mathrm{T}$ is mutually interpretable with Robinson's $\mathrm{Q}$.

The Axioms of $\mathrm{R}$

$\mathrm{R}_{1} \bar{n}+\bar{m}=\overline{n+m} ; \quad \mathrm{R}_{2} \bar{n} \times \bar{m}=\overline{n m} ; \quad \mathrm{R}_{3} \bar{n} \neq \bar{m}$ for $n \neq m ;$

$\mathrm{R}_{4} \forall x[x \leq \bar{n} \rightarrow x=0 \vee \ldots \vee x=\bar{n}] ; \quad \mathrm{R}_{5} \quad \forall x[x \leq \bar{n} \vee \bar{n} \leq x]$

\section{The Axioms of $Q$}

$\mathrm{Q}_{1} \forall x y[S x=S y \rightarrow x=y] ; \quad \mathrm{Q}_{2} \forall x[S x \neq 0] ; \quad \mathrm{Q}_{3} \forall x[x \neq 0 \rightarrow \exists y[x=S y]] ;$

$\mathrm{Q}_{4} \forall x[x+0=x] ; \quad \mathrm{Q}_{5} \forall x y[x+S y=S(x+y)] ; \quad \mathrm{Q}_{6} \forall x[x \times 0=0] ;$

$\mathrm{Q}_{7} \forall x y[x \times S y=(x \times y)+x] ; \quad \mathrm{Q}_{8} \quad \forall x y[x \leq y \leftrightarrow \exists z[x+z=y]]$

Fig. 1. The axioms of $\mathrm{R}$ are given by axiom schemes where $n, m \in \mathbb{N}$ and $\bar{n}$ denotes the $n^{\text {th }}$ numeral, that is, $\overline{0} \equiv 0$ and $\overline{n+1} \equiv S \bar{n}$.

The seminal theories $\mathrm{R}$ and $\mathrm{Q}$ are theories of arithmetic. The theory $\mathrm{R}$ is given by axiom schemes, and $Q$ is a finitely axiomatizable extension of $R$, see Fig. 1 ( $Q$ is also known as Robinson arithmetic and is more or less Peano arithmetic without the induction scheme). It was proved in Tarski et al. [9] that $\mathrm{R}$ and $\mathrm{Q}$ are essentially undecidable. Another seminal essentially undecidable first-order theory is Grzegorcyk's TC. This is a theory of concatenation. The language is $\{*, \alpha, \beta\}$ where $\alpha$ and $\beta$ are constant symbols and $*$ is a binary function symbol. The standard TC model is the structure where the universe is $\{a, b\}^{+}$(all finite nonempty strings over the alphabet $\{a, b\}), *$ is concatenation, $\alpha$ is the string $a$ and $\beta$ is the string $b$. It was proved in Grzegorzyk and Zdanowski [3] that TC is essentially undecidable. It was later proved that TC is mutually interpretable with Q, see Visser [10] for further references. The theory WTC $^{-\epsilon}$ is a weaker variant of TC that has been shown to be mutually interpretable with $R$, see Higuchi and Horihata [4] for more details and further references. The axioms of TC and WTC ${ }^{-\epsilon}$ can be found in Fig. 2.

The overall picture shows three finitely axiomatizable and essentially undecidable first-order theories of different character and nature: $Q$ is a theory of arithmetic, TC is a theory of concatenation, and $\mathrm{T}$ is a theory of terms (it may also be viewed as a theory of binary trees). All three theories are mutually interpretable with each other, and each of them come with a weaker variant given by axiom schemes. These weaker variants are also essentially undecidable and mutually interpretable with each other.

The theory $\mathrm{T}$ has, in contrast to $\mathrm{Q}$ and $\mathrm{TC}$, a purely universal axiomatization, that is, there are no occurrences of existential quantifiers in the axioms. Moreover, its weaker variant WT has a neat and very compact axiomatization compared to $\mathrm{R}$ and $\mathrm{WTC}^{-\epsilon}$. 


$$
\begin{aligned}
& \mathrm{WTC}_{1}^{-\epsilon} \forall x y z[(x *(y * z) \sqsubseteq \underline{t} \vee(x * y) * z \sqsubseteq \underline{t}) \rightarrow x *(y * z)=(x * y) * z] ; \\
& \mathrm{WTC}_{2}^{-\epsilon} \forall x y z u[x * y=z * u \wedge x * y \sqsubseteq \underline{t} \rightarrow((x=z \wedge y=u) \vee \\
& \exists w[(x * w=z \wedge w * u=y) \vee(z * w=x \wedge w * y=u])] \\
& \mathrm{WTC}_{3}^{-\epsilon} \forall x y[\alpha \neq x * y] ; \quad \mathrm{WTC}_{4}^{-\epsilon} \forall x y[\beta \neq x * y] ; \quad \mathrm{WTC}_{5}^{-\epsilon} \alpha \neq \beta
\end{aligned}
$$

where $x \sqsubseteq y$ is defined by

$$
x=y \vee \exists z_{1} z_{2}\left[z_{1} * x=y \vee x * z_{2}=y \vee\left(z_{1} * x\right) * z_{2}=y \vee z_{1} *\left(x * z_{2}\right)=y\right] .
$$

The Axioms of TC

$$
\begin{aligned}
& \mathrm{TC}_{1} \forall x y z[x *(y * z)=(x * y) * z] \\
& \mathrm{TC}_{2} \forall x y z u[x * y=z * u \rightarrow((x=z \wedge y=u) \vee \\
& \exists w[(x * w=z \wedge w * u=y) \vee(z * w=x \wedge w * y=u])] ; \\
& \mathrm{TC}_{3} \forall x y[\alpha \neq x * y] ; \quad \mathrm{TC}_{4} \forall x y[\beta \neq x * y] ; \quad \operatorname{TC}_{5} \alpha \neq \beta
\end{aligned}
$$

Fig. 2. $\mathrm{WTC}_{1}^{-\epsilon}$ and $\mathrm{WTC}_{2}^{-\epsilon}$ are axiom schemes where $t \in\{a, b\}^{+}$and $\underline{t}$ is a term inductively defined by: $\underline{a} \equiv \alpha, \underline{b} \equiv \beta, \underline{a u} \equiv \alpha * \underline{u}$ and $\underline{b u} \equiv \beta * \underline{u}$.

Another interesting theory which is known to be mutually interpretable with $\mathrm{Q}$, and thus also with TC and $\mathrm{T}$, is the adjunctive set theory AST. More on AST and adjunctive set theory can found in Damnjanovic [2]. For recent results related to the work in the present paper, we refer the reader to Jerabek [5], Cheng [1] and Kristiansen and Murwanashyaka [7].

The rest of this paper is fairly technical, and we will assume that the reader is familiar with first-order theories and the interpretation techniques introduced in Tarski et al. [9]. In Sect. 2 we prove that R and WT are mutually interpretable. In Sect. 3 we prove that $Q$ is interpretable in $T$. We expect that $T$ can be interpreted in $Q$ by standard techniques available in the literature.

\section{$2 \quad \mathrm{R}$ and WT Are Mutually Interpretable}

The theory $\mathrm{R}^{-}$over the language of Robinson arithmetic is given by the axiom schemes

$$
\begin{aligned}
& \mathrm{R}_{1}^{-} \bar{n}+\bar{m}=\overline{n+m} ; \quad \mathrm{R}_{2}^{-} \bar{n} \times \bar{m}=\overline{n m} ; \quad \mathrm{R}_{3}^{-} \bar{n} \neq \bar{m} \text { for } n \neq m \\
& \mathrm{R}_{4}^{-} \quad \forall x[x \leq \bar{n} \leftrightarrow x=0 \vee \ldots \vee x=\bar{n}]
\end{aligned}
$$

where $n, m \in \mathbb{N}$. Recall that $\bar{n}$ denotes the $n^{\text {th }}$ numeral, that is, $\overline{0} \equiv 0$ and $\overline{n+1} \equiv S \bar{n}$.

We now proceed to interpret $\mathrm{R}^{-}$in WT. We choose the domain $I(x) \equiv x=x$ (thus we can just ignore the domain). Furthermore, we translate the successor function $S(x)$ as the function given by $\lambda x .\langle x, \perp\rangle$, and we translate the constant 0 as $\langle\perp, \perp\rangle$. Let $\bar{n}^{\star}$ denote the translation of the numeral $\bar{n}$. Then we have $\overline{n+1}^{\star} \equiv\left\langle\bar{n}^{\star}, \perp\right\rangle$. It follows from $\mathrm{WT}_{1}$ that the translation of each instance of $\mathrm{R}_{3}^{-}$is a theorem of WT since $\bar{m}^{\star}$ and $\bar{n}^{\star}$ are different terms whenever $m \neq n$. 
We translate $x \leq y$ as $x \sqsubseteq y \wedge x \neq \perp$. It is easy to see that

$$
\mathrm{W \top} \vdash \forall x\left[x \sqsubseteq \bar{n}^{\star} \wedge x \neq \perp \leftrightarrow \bigvee_{s \in \mathcal{T}(n)} x=s\right]
$$

where $\mathcal{T}(n)=\mathcal{S}\left(\bar{n}^{\star}\right) \backslash\{\perp\}$ and $\mathcal{S}\left(\bar{n}^{\star}\right)$ denotes the set of all subterms of $\bar{n}^{\star}$. We observe that $\mathcal{T}(n)=\left\{\bar{k}^{\star} \mid k \leq n\right\}$ and that (1) indeed is the translation of the axiom scheme $\mathrm{R}_{4}^{-}$. Hence we conclude that the translation of each instance of $\mathrm{R}_{4}^{-}$is a theorem of WT.

Next we discuss the translation of + . The idea is to obtain $n+i$ through a formation sequence of length $i$. Such a sequence will be represented by a term of the form

$$
\left\langle\ldots\left\langle\left\langle\left\langle\bar{n}^{\star}, \overline{0}^{\star}\right\rangle,\left\langle\overline{n+1}^{\star}, \overline{1}^{\star}\right\rangle\right\rangle,\left\langle\overline{n+2}^{\star}, \overline{2}^{\star}\right\rangle\right\rangle \ldots,\left\langle\overline{n+i^{\star}}, \bar{i}^{\star}\right\rangle\right\rangle .
$$

Accordingly we translate $x+y=z$ by the predicate $\operatorname{add}(x, y, z)$ given by the formula

$$
\begin{aligned}
\left(y=\overline{0}^{\star} \wedge z=x\right) \vee\left\{y \neq \overline{0}^{\star} \wedge \exists W\left[\left\langle x, \overline{0}^{\star}\right\rangle \sqsubseteq W \wedge\right.\right. \\
\forall X \forall Y \sqsubseteq y[\langle X, Y\rangle \sqsubseteq W \wedge Y \neq y \wedge Y \neq \perp \rightarrow \\
(\langle\langle X, \perp\rangle,\langle Y, \perp\rangle\rangle \sqsubseteq W \wedge(\langle Y, \perp\rangle=y \rightarrow\langle X, \perp\rangle=z))]]\} .
\end{aligned}
$$

Lemma 1. For any $m, n \in \mathbb{N}$, we have

$$
\mathrm{WT} \vdash \forall z\left[\operatorname{add}\left(\bar{n}^{\star}, \bar{m}^{\star}, z\right) \leftrightarrow z=\overline{n+m}^{\star}\right] .
$$

Proof. First we prove that WT $\vdash \operatorname{add}\left(\bar{n}^{\star}, \bar{m}^{\star}, \overline{n+m}^{\star}\right)$. This is obvious if $m=0$. Assume $m>0$. Let

$$
S_{0}^{n} \equiv\left\langle\bar{n}^{\star}, \overline{0}^{\star}\right\rangle \quad \text { and } \quad S_{i+1}^{n} \equiv\left\langle S_{i}^{n},\left\langle\overline{n+i+1}{ }^{\star}, \overline{i+1}{ }^{\star}\right\rangle\right\rangle
$$

and observe that $S_{i}^{n}$ is of the form (2). We will argue that we can choose the $W$ in the definition of $\operatorname{add}(x, y, z)$ to be the term $S_{m}^{n}$.

So let $W=S_{m}^{n}$. By the axioms of WT, we have $\left\langle\bar{n}^{\star}, \overline{0}^{\star}\right\rangle \sqsubseteq W$. Assume

$$
\langle X, Y\rangle \sqsubseteq W \text { and } Y \neq y=\bar{m}^{\star} \text { and } Y \sqsubseteq y=\bar{m}^{\star} \text { and } Y \neq \perp \text {. }
$$

By the axioms of WT, we have that $Y \sqsubseteq \bar{m}^{\star}, Y \neq \bar{m}^{\star}$ and $Y \neq \perp$ imply $Y=\bar{k}^{\star}$ for some $k<m$. Since $\langle X, Y\rangle \sqsubseteq W$, we know by $\mathrm{WT}_{2}$ that $\langle X, Y\rangle$ is one of the subterms of $W$. By $\mathrm{WT}_{1}$ and the form of $S_{m}^{n}$, we conclude that $X=\overline{n+k}^{\star}$. Furthermore, the form of $S_{m}^{n}$ and $\mathrm{WT}_{2}$ then ensures that $\langle\langle X, \perp\rangle,\langle Y, \perp\rangle\rangle \sqsubseteq$ $W=S_{m}^{n}$. Moreover, if $\langle Y, \perp\rangle=\bar{m}^{\star}$, then by $\mathrm{WT}_{1}$, we must have $k=m-1$, and thus, $\langle X, \perp\rangle=\left\langle\overline{n+(m-1)}{ }^{\star}, \perp\right\rangle=\overline{n+m}^{\star}$. This proves that we can deduce add $\left(\bar{n}^{\star}, \bar{m}^{\star}, \overline{n+m}^{\star}\right)$ from the axioms of WT, and thus we also have

$$
\mathrm{WT} \vdash \forall z\left[z=\overline{n+m}^{\star} \rightarrow \operatorname{add}\left(\bar{n}^{\star}, \bar{m}^{\star}, z\right)\right] .
$$


Next we prove that the converse implication add $\left(\bar{n}^{\star}, \bar{m}^{\star}, z\right) \rightarrow z=\overline{n+m}^{\star}$ follows from the axioms of WT (and thus the lemma follows). This is obvious when $m=0$. Assume $m \neq 0$ and $\operatorname{add}\left(\bar{n}^{\star}, \bar{m}^{\star}, z\right)$. Then we have $W$ such that $\left\langle\bar{n}^{\star}, \overline{0}^{\star}\right\rangle \sqsubseteq W$ and

$$
\begin{aligned}
\forall X \forall Y \sqsubseteq \bar{m}^{\star}\left[\langle X, Y\rangle \sqsubseteq W \wedge Y \neq \bar{m}^{\star} \wedge Y \neq \perp \quad \rightarrow\right. \\
\left.\left(\langle\langle X, \perp\rangle,\langle Y, \perp\rangle\rangle \sqsubseteq W \wedge\left(\langle Y, \perp\rangle=\bar{m}^{\star} \rightarrow\langle X, \perp\rangle=z\right)\right)\right] .
\end{aligned}
$$

Since $\left\langle n, \overline{0}^{\star}\right\rangle \sqsubseteq W$ and (3) hold, we have $\left\langle\overline{n+k+1}^{\star}, \overline{k+1}^{\star}\right\rangle \sqsubseteq W$ for any $k<m$. It also follows from (3) that $z=\overline{n+k+1}{ }^{\star}$ when $m=k+1$.

It follows from the preceding lemma that there for any $n, m \in \mathbb{N}$ exists a unique $k \in \mathbb{N}$ such that WT $\vdash$ add $\left(\bar{n}^{\star}, \bar{m}^{\star}, \bar{k}^{\star}\right)$. We translate $x+y=z$ by the predicate $\phi_{+}$where $\phi_{+}(x, y, z)$ is the formula

$$
(\exists ! u[\operatorname{add}(x, y, u)] \wedge \operatorname{add}(x, y, z)) \vee(\neg \exists ! u[\operatorname{add}(x, y, u)] \wedge z=\perp) .
$$

The second disjunct of (4) ensures the functionality of our translation, that is, it ensures that WT $\vdash \forall x y \exists ! x \phi_{+}(x, y, z)$ (the same technique is used in [6]). By Lemma 1 , we have $\mathrm{WT} \vdash \phi_{+}\left(\bar{n}^{\star}, \bar{m}^{\star}, \overline{n+m}{ }^{\star}\right)$. This shows that the translation of any instance of the axiom scheme $\mathrm{R}_{1}^{-}$can be deduced from the axioms of WT.

We can also achieve a translation of $x \times y=z$ such that the translation of each instance of $\mathrm{R}_{2}^{-}$can be deduced from the axioms of WT. Such a translation claims the existence of a term $S_{m}^{n}$ where

$$
S_{1}^{n} \equiv\left\langle\bar{n}^{\star}, \overline{1}^{\star}\right\rangle \quad \text { and } \quad S_{i+1}^{n} \equiv\left\langle S_{i}^{n},\left\langle\overline{(i+1) n}{ }^{\star}, \overline{i+1}^{\star}\right\rangle\right\rangle
$$

and will more or less be based on the same ideas as our translation of $x+y=z$. We omit the details.

\section{Theorem 2. R and WT are mutually interpretable.}

Proof. We have seen how to interpret $\mathrm{R}^{-}$in WT. It follows straightforwardly from results proved in Jones and Shepherdson [6] that $\mathrm{R}^{-}$and $\mathrm{R}$ are mutually interpretable. Thus R is interpretable in WT. A result of Visser [11] states that a theory is interpretable in $\mathrm{R}$ if and only if it is locally finitely satisfiable, that is, each finite subset of the non-logical axioms has a finite model. Since WT clearly is locally finitely satisfiable, WT is interpretable in R.

\section{$3 \quad \mathbf{Q}$ is Interpretable in $\mathbf{T}$}

The language of the arithmetical theory $\mathrm{Q}^{-}$is $\{0, S, M, A\}$ where 0 is a constant symbol, $S$ is a unary function symbol, and $A$ and $M$ are ternary predicate symbols. The non-logical axioms of the first-order theory $\mathrm{Q}^{-}$are the the following:

$\mathrm{A} \forall x y z_{1} z_{2}\left[A\left(x, y, z_{1}\right) \wedge A\left(x, y, z_{2}\right) \rightarrow z_{1}=z_{2}\right]$;

$\mathrm{M} \forall x y z_{1} z_{2}\left[M\left(x, y, z_{1}\right) \wedge M\left(x, y, z_{2}\right) \rightarrow z_{1}=z_{2}\right]$;

$\mathrm{Q}_{1} \forall x y[x \neq y \rightarrow S x \neq S y] ; \quad \mathrm{Q}_{2} \forall x[S x \neq 0] ; \quad \mathrm{Q}_{3} \forall x[x=0 \vee \exists y[x=S y]]$;

$\mathrm{G}_{4} \forall x[A(x, 0, x)] ; \quad \mathrm{G}_{5} \forall x y u[\exists z[A(x, y, z) \wedge u=S z] \rightarrow A(x, S y, u)]$;

$\mathrm{G}_{6} \forall x[M(x, 0,0)] ; \quad \mathrm{G}_{7} \forall x y u[\exists z[M(x, y, z) \wedge A(z, x, u)] \rightarrow M(x, S y, u)]$. 
Svejdar [8] proved that $\mathrm{Q}^{-}$and $\mathrm{Q}$ are mutually interpretable. We will prove that $\mathrm{Q}^{-}$is interpretable in $\mathrm{T}$.

The first-order theory $\mathrm{T}^{+}$is $\mathrm{T}$ extended by the two non-logical axioms

$$
\mathrm{T}_{5} \forall x[x \sqsubseteq x] \quad \text { and } \quad \mathrm{T}_{6} \forall x y z[x \sqsubseteq y \wedge y \sqsubseteq z \rightarrow x \sqsubseteq z] .
$$

Lemma 3. $\mathrm{T}^{+}$is interpretable in $\mathrm{T}$.

Proof. We simply relativize quantification to the domain

$$
I=\{x \mid x \sqsubseteq x \wedge \forall u v[u \sqsubseteq v \wedge v \sqsubseteq x \rightarrow u \sqsubseteq x]\} .
$$

Suppose $x_{1}, x_{2} \in I$. We show that $\left\langle x_{1}, x_{2}\right\rangle \in I$. Since $\left\langle x_{1}, x_{2}\right\rangle=\left\langle x_{1}, x_{2}\right\rangle$, we have $\left\langle x_{1}, x_{2}\right\rangle \sqsubseteq\left\langle x_{1}, x_{2}\right\rangle$ by $\mathrm{T}_{4}$. Suppose now that $u \sqsubseteq v \wedge v \sqsubseteq\left\langle x_{1}, x_{2}\right\rangle$. We need to show that $u \sqsubseteq\left\langle x_{1}, x_{2}\right\rangle$. By $\mathrm{T}_{4}$ and $v \sqsubseteq\left\langle x_{1}, x_{2}\right\rangle$, at least one of the following three cases holds: (a) $v=\left\langle x_{1}, x_{2}\right\rangle$, (b) $v \sqsubseteq x_{1}$, (c) $v \sqsubseteq x_{2}$. Case (a): Since $u \sqsubseteq v$ and $v=\left\langle x_{1}, x_{2}\right\rangle$, we have $u \sqsubseteq\left\langle x_{1}, x_{2}\right\rangle$ by our logical axioms. Case (b): $u \sqsubseteq v \wedge v \sqsubseteq x_{1}$ implies $u \sqsubseteq x_{1}$ since $x_{1} \in I$. By $\mathrm{T}_{4}$, we have $u \sqsubseteq\left\langle x_{1}, x_{2}\right\rangle$. Case (c): We have $u \sqsubseteq\left\langle x_{1}, x_{2}\right\rangle$ by an argument symmetric to the one used in Case (b). Hence, $\forall u v\left[u \sqsubseteq v \wedge v \sqsubseteq\left\langle x_{1}, x_{2}\right\rangle \rightarrow u \sqsubseteq\left\langle x_{1}, x_{2}\right\rangle\right]$.

This proves that $I$ is closed under $\langle\cdot, \cdot\rangle$. It follows from $\mathrm{T}_{3}$ that $\perp \in I$, and thus $I$ satisfies the domain condition. Clearly, the translation of each non-logical axiom of $\mathrm{T}^{+}$is a theorem of $\mathrm{T}$.

We now proceed to interpret $\mathrm{Q}^{-}$in $\mathrm{T}^{+}$. We choose the domain $N$ given by

$$
N(x) \equiv x \neq \perp \wedge \forall y \sqsubseteq x[y=\perp \vee \exists z[y=\langle z, \perp\rangle]] .
$$

Lemma 4. We have (i) $\mathrm{T}^{+} \vdash N(\langle\perp, \perp\rangle)$, (ii) $\mathrm{T}^{+} \vdash \forall x[N(x) \rightarrow N(\langle x, \perp\rangle)]$ and (iii) $\mathrm{T}^{+} \vdash \forall y z[N(y) \wedge z \sqsubseteq y \rightarrow(z=\perp \vee N(z))]$.

Proof. It follows from $\mathrm{T}_{1}, \mathrm{~T}_{3}$ and $\mathrm{T}_{4}$ that (i) holds. In order to see that (ii) holds, assume $N(x)$ (we will argue that $N(\langle x, \perp\rangle)$ holds). Suppose $y \sqsubseteq\langle x, \perp\rangle$. Now, $N(\langle x, \perp\rangle)$ follows from

$$
y=\perp \vee \exists z[y=\langle z, \perp\rangle] .
$$

Thus it is sufficient to argue that (5) holds. By $\mathrm{T}_{4}$, we know that $y \sqsubseteq\langle x, \perp\rangle$ implies $y=\langle x, \perp\rangle \vee y \sqsubseteq x \vee y \sqsubseteq \perp$. The case $y=\langle x, \perp\rangle$ : We obviously have $\exists z[y=\langle z, \perp\rangle]$ and thus (5) holds. The case $y \sqsubseteq x$ : (5) holds since $N(x)$ holds. The case $y \sqsubseteq \perp$ : We have $y=\perp$ by $\mathrm{T}_{3}$, and thus (5) holds. This proves (ii).

We turn to the proof of (iii). Suppose $N(y) \wedge z \sqsubseteq y$ (we show $z=\perp \vee N(z)$ ). Assume $w \sqsubseteq z$. By $\mathrm{T}_{6}$, we have $w \sqsubseteq y$, moreover, since $N(y)$ holds, we have $w=\perp \vee \exists u[w=\langle u, \perp\rangle]$. Thus, we conclude that

$$
\forall w \sqsubseteq z[w=\perp \vee \exists u[w=\langle u, \perp\rangle]] .
$$

Now

$$
z=\perp \vee \underbrace{(z \neq \perp \wedge \forall w \sqsubseteq z[w=\perp \vee \exists u[w=\langle u, \perp\rangle]])}_{N(z)}
$$

follows tautologically from (6). 
We interpret 0 as $\langle\perp, \perp\rangle$. We interpret the successor function $S x$ as $\lambda x .\langle x, \perp\rangle$. To improve the readability we will occasionally write $\dot{0}$ in place of $\langle\perp, \perp\rangle, \dot{\mathrm{S}} t$ in place of $\langle t, \perp\rangle$ and $t \in N$ in place of $N(t)$. We will also write $\exists x \in N[\eta]$ and $\forall x \in N[\eta]$ in place of, respectively, $\exists x[N(x) \wedge \eta]$ and $\forall x[N(x) \rightarrow \eta]$. Furthermore, $\mathbf{Q} x_{1}, \ldots, x_{n} \in N$ is shorthand for $\mathbf{Q} x_{1} \in N \ldots \mathbf{Q} x_{n} \in N$ where $\mathbf{Q}$ is either $\forall$ or $\exists$.

Lemma 5. The translations of $\mathrm{Q}_{1}, \mathrm{Q}_{2}$ and $\mathrm{Q}_{3}$ are theorems of $\mathrm{T}^{+}$.

Proof. The translation of $\mathrm{Q}_{1}$ is $\forall x, y \in N[x \neq y \rightarrow \dot{\mathrm{S}} x \neq \dot{\mathrm{S}} y]$. By $\mathrm{T}_{2}$, we have $x \neq y \rightarrow \dot{\mathrm{S}} x \neq \dot{\mathrm{S}} y$ for any $x, y$, and thus, the translation of $\mathrm{Q}_{1}$ is a theorem of $\mathrm{T}^{+}$.

The translation of $\mathrm{Q}_{2}$ is $\forall x \in N[\dot{\mathrm{S}} x \neq \dot{0}]$. Assume $x \in N$. Then we have $x \neq \perp$, and by $\mathrm{T}_{2}$, we have $\dot{\mathrm{S}} n \equiv\langle x, \perp\rangle \neq\langle\perp, \perp\rangle \equiv \dot{0}$.

The translation of $\mathrm{Q}_{3}$ is $\forall x \in N[x=\dot{0} \vee \exists y \in N[x=\dot{\mathrm{S}} y]]$. Assume $x \in N$, that is, assume

$$
x \neq \perp \wedge \forall y \sqsubseteq x[y=\perp \vee \exists z[y=\langle z, \perp\rangle]] .
$$

By $\mathrm{T}_{5}$, we have $x \sqsubseteq x$. By $(7)$ and $x \sqsubseteq x$, we have

$$
x \neq \perp \wedge(x=\perp \vee \exists z[x=\langle z, \perp\rangle])
$$

and then, by a tautological inference, we also have $\exists z[x=\langle z, \perp\rangle]$. Thus, we have $z$ such that $\langle z, \perp\rangle \equiv \dot{\mathrm{S}} z=x \in N$. By Lemma 4 (iii), we have $z=\perp \vee z \in N$. If $z=\perp$, we have $x=\langle\perp, \perp\rangle \equiv \dot{0}$. If $z \in N$, we have $z \in N$ such that $x=\dot{\mathrm{S}} z$. Thus, $\mathrm{T}^{+} \vdash \forall x \in N[x=\dot{0} \vee \exists y \in N[x=\dot{\mathrm{S}} y]]$.

Before we give the translation of $A$, we will provide some intuition. The predicate $A(a, b, c)$ holds in the standard model for $\mathrm{Q}^{-}$iff $a+b=c$. Let $\widetilde{0} \equiv \dot{0}$ and $\widetilde{n+1} \equiv \dot{\mathrm{S}} \widetilde{n}$, and observe that $a+b=c$ iff there exists an $\mathcal{L}_{\mathrm{T}}$-term of the form

$$
\langle\ldots\langle\langle\langle\perp,\langle\widetilde{a}, \widetilde{0}\rangle\rangle,\langle\widetilde{a+1}, \widetilde{1}\rangle\rangle,\langle\widetilde{a+2}, \widetilde{2}\rangle\rangle \ldots,\langle\widetilde{a+b}, \widetilde{b}\rangle\rangle
$$

where $c=a+b$. We will give a predicate $\phi_{A}$ such that $\phi_{A}(\widetilde{a}, \widetilde{b}, w)$ holds in $\mathrm{T}^{+}$ iff $w$ is of the form (8). Thereafter we will use $\phi_{A}$ to give the translation $\Psi_{A}$ of A.

Let $\phi_{A}(x, y, w) \equiv$

$$
\begin{aligned}
(y=\dot{0} \rightarrow w=\langle\perp,\langle x, \dot{0}\rangle\rangle) \wedge \exists w^{\prime} \exists z \in N\left[w=\left\langle w^{\prime},\langle z, y\rangle\right\rangle\right] \wedge \\
\forall u \forall Y, Z \in N\left[\theta_{A}(u, w, Y, Z)\right]
\end{aligned}
$$

where $\theta_{A}(u, w, Y, Z) \equiv$

$$
\begin{aligned}
&\langle u,\langle Z, Y\rangle\rangle \sqsubseteq w \wedge Y \neq \dot{0} \rightarrow \\
& \exists v \exists Y^{\prime} Z^{\prime} \in N\left[Z=\dot{\mathrm{S}} Z^{\prime} \wedge Y=\dot{\mathrm{S}} Y^{\prime} \wedge u=\left\langle v,\left\langle Z^{\prime}, Y^{\prime}\right\rangle\right\rangle \wedge\right. \\
&\left.\quad\left(Y^{\prime}=\dot{0} \rightarrow\left(Z^{\prime}=x \wedge v=\perp\right)\right)\right] .
\end{aligned}
$$


The translation $\Psi_{A}$ of $A$ is $\Psi_{A}(x, y, z) \equiv$

$$
\exists w\left[\phi_{A}(x, y, w) \wedge \exists w^{\prime}\left[w=\left\langle w^{\prime},\langle z, y\rangle\right\rangle\right] \wedge \forall u\left[\phi_{A}(x, y, u) \rightarrow u=w\right]\right] .
$$

\section{Lemma 6.}

$$
\mathrm{T}^{+} \vdash \forall x \in N \forall w\left[\phi_{A}(x, \dot{0}, w) \leftrightarrow w=\langle\perp,\langle x, \dot{0}\rangle\rangle\right] .
$$

Proof. We assume $x \in N$ and prove the equivalence

$$
\phi_{A}(x, \dot{0}, w) \leftrightarrow w=\langle\perp,\langle x, \dot{0}\rangle\rangle
$$

The left-right direction of (9) follows straightforwardly from the definition of $\phi_{A}$. To prove the right-left implication of (9), we need to prove $\phi_{A}(x, \dot{0},\langle\perp,\langle x, \dot{0}\rangle\rangle)$. It is easy to see that $\phi_{A}(x, \dot{0},\langle\perp,\langle x, \dot{0}\rangle\rangle)$ holds if

$$
\forall u \forall Y, Z \in N\left[\theta_{A}(u,\langle\perp,\langle x, \dot{0}\rangle\rangle, Y, Z)\right]
$$

holds, and to show (10), it suffices to show that

$$
x, Y, Z \in N \text { and }\langle u,\langle Z, Y\rangle\rangle \sqsubseteq\langle\perp,\langle x, \dot{0}\rangle\rangle \text { and } Y \neq \dot{0}
$$

is a contradiction. (If (11) is a contradiction, then (10) will hold as the antecedent of $\theta_{A}$ will be false for all $x, Y, Z \in N$ and all $u$.)

By $\mathrm{T}_{4}$ and $\langle u,\langle Z, Y\rangle\rangle \sqsubseteq\langle\perp,\langle x, \dot{0}\rangle\rangle$ we have to deal with the following three cases: (a) $\langle u,\langle Z, Y\rangle\rangle=\langle\perp,\langle x, \dot{0}\rangle\rangle$, (b) $\langle u,\langle Z, Y\rangle\rangle \sqsubseteq \perp$ and (c) $\langle u,\langle Z, Y\rangle\rangle \sqsubseteq$ $\langle x, \dot{0}\rangle$. Case: (a): We have $Y=\dot{0}$ by $\mathrm{T}_{2}$, but we have $Y \neq \dot{0}$ in (11). Case (b): We have $\langle u,\langle Z, Y\rangle\rangle=\perp$ by $\mathrm{T}_{3}$, and this contradicts $\mathrm{T}_{1}$. Case (c): By $\mathrm{T}_{4}$, this case splits into the three subcases: (a') $\langle u,\langle Z, Y\rangle\rangle=\langle x, \dot{0}\rangle,\left(\mathrm{b}^{\prime}\right)\langle u,\langle Z, Y\rangle\rangle \sqsubseteq x$ and $\left(\mathrm{c}^{\prime}\right)\langle u,\langle Z, Y\rangle\rangle \sqsubseteq \dot{0}$. Case (a'): We have $\langle u,\langle Z, Y\rangle\rangle=\langle x,\langle\perp, \perp\rangle\rangle$ since $\dot{0}$ is shorthand for $\langle\perp, \perp\rangle$. Thus, by $\mathrm{T}_{2}$, we have $Z=\perp$ and $Y=\perp$. This contradicts $Y, Z \in N$. Case (b'): We have $\langle u,\langle Z, Y\rangle\rangle \sqsubseteq x$ and $x \in N$. By Lemma 4 (iii), we have $\langle u,\langle Z, Y\rangle\rangle=\perp$ or $\langle u,\langle Z, Y\rangle\rangle \in N$. Now, $\langle u,\langle Z, Y\rangle\rangle=\perp$ contradicts $\mathrm{T}_{1}$. Furthermore, by our definitions, $\langle u,\langle Z, Y\rangle\rangle \in N$ implies that

$$
\forall y_{0} \sqsubseteq\langle u,\langle Z, Y\rangle\rangle\left[y_{0}=\perp \vee \exists z_{0}\left[y_{0}=\left\langle z_{0}, \perp\right\rangle\right]\right] .
$$

By $\mathrm{T}_{5}$, we have $\langle u,\langle Z, Y\rangle\rangle=\perp \vee \exists z_{0}\left[\langle u,\langle Z, Y\rangle\rangle=\left\langle z_{0}, \perp\right\rangle\right]$, and this yields a contradiction together with $\mathrm{T}_{1}$ and $\mathrm{T}_{2}$. Case (c') is similar to Case (a'), but a bit simpler. This completes the proof of the lemma.

\section{Lemma 7.}

$$
\begin{array}{r}
\mathrm{T}^{+} \vdash \forall x, y \in N \forall z w w^{\prime}\left[w=\left\langle w^{\prime},\langle z, y\rangle\right\rangle \wedge \phi_{A}(x, y, w) \rightarrow\right. \\
\left.\quad \phi_{A}(x, \dot{\mathrm{S}} y,\langle w,\langle\dot{\mathrm{S}} z, \dot{\mathrm{S}} y\rangle\rangle)\right] .
\end{array}
$$

Proof. We assume

$$
x, y \in N \text { and } w=\left\langle w^{\prime},\langle z, y\rangle\right\rangle \text { and } \phi_{A}(x, y, w) .
$$


We need to prove $\phi_{A}(x, \dot{\mathrm{S}} y,\langle w,\langle\dot{\mathrm{S}} z, \dot{\mathrm{S}} y\rangle\rangle) \equiv$

$$
\begin{aligned}
&(\dot{\mathrm{S}} y=\dot{0} \rightarrow w=\langle\perp,\langle x, \dot{0}\rangle\rangle) \wedge \\
& \exists w_{0} \exists z_{0} \in N\left[\langle w,\langle\dot{\mathrm{S}} z, \dot{\mathrm{S}} y\rangle\rangle=\left\langle w_{0},\left\langle z_{0}, \dot{\mathrm{S}} y\right\rangle\right\rangle\right] \wedge \\
& \quad \forall u \forall Y, Z \in N\left[\theta_{A}(u,\langle w,\langle\dot{\mathrm{S}} z, \dot{\mathrm{S}} y\rangle\rangle, Y, Z)\right]
\end{aligned}
$$

First we prove

$$
z \in N \quad \text { and } \quad \dot{\mathrm{S}} z \in N
$$

Since $\phi_{A}(x, y, w)$ holds by our assumptions (12), we have $z_{1} \in N$ and $w_{1}$ such that $w=\left\langle w_{1},\left\langle z_{1}, y\right\rangle\right\rangle$. We have also assumed $w=\left\langle w^{\prime},\langle z, y\rangle\right\rangle$. By $\mathrm{T}_{2}$, we have $z=z_{1}$, and thus $z \in N$. By Lemma 4 (ii), we have $\mathrm{S} z \in N$. This proves (14).

The second conjunct of (13) follows straightforwardly from (14). (simply let $z_{0}$ be $\dot{\mathrm{S}} z$ and let $w_{0}$ be $\left.w\right)$. The first conjunct follows easily from $\mathrm{T}_{2}$ and the assumption $y \in N$. Thus, we are left to prove the third conjunct of (13), namely

$$
\begin{aligned}
\forall u \forall Y, Z \in N[\langle u,\langle Z, Y\rangle\rangle \sqsubseteq\langle w,\langle\dot{\mathrm{S}} z, \dot{\mathrm{S}} y\rangle\rangle \wedge Y \neq \dot{0} \rightarrow \\
\exists v \exists Y^{\prime} Z^{\prime} \in N\left[Z=\dot{\mathrm{S}} Z^{\prime} \wedge Y=\dot{\mathrm{S}} Y^{\prime} \wedge u=\left\langle v,\left\langle Z^{\prime}, Y^{\prime}\right\rangle\right\rangle \wedge\right. \\
\left.\left.\left(Y^{\prime}=\dot{0} \rightarrow\left(Z^{\prime}=x \wedge v=\perp\right)\right)\right]\right]
\end{aligned}
$$

In order to do so, we assume

$$
Y, Z \in N \text { and }\langle u,\langle Z, Y\rangle\rangle \sqsubseteq\langle w,\langle\dot{\mathrm{S}} z, \dot{\mathrm{S}} y\rangle\rangle \text { and } Y \neq \dot{0}
$$

and prove

$$
\begin{aligned}
\exists v \exists Y^{\prime} Z^{\prime} \in N\left[Z=\dot{\mathrm{S}} Z^{\prime} \wedge Y=\dot{\mathrm{S}} Y^{\prime} \wedge u=\left\langle v,\left\langle Z^{\prime}, Y^{\prime}\right\rangle\right\rangle \wedge\right. \\
\left.\quad\left(Y^{\prime}=\dot{0} \rightarrow\left(Z^{\prime}=x \wedge v=\perp\right)\right)\right] .
\end{aligned}
$$

By our assumptions (16), we have $\langle u,\langle Z, Y\rangle\rangle \sqsubseteq\langle w,\langle\dot{\mathrm{S}} z, \dot{\mathrm{S}} y\rangle\rangle$, and then $\mathrm{T}_{4}$ yields three cases: (a) $\langle u,\langle Z, Y\rangle\rangle=\langle w,\langle\dot{\mathrm{S}} z, \dot{\mathrm{S}} y\rangle\rangle$, (b) $\langle u,\langle Z, Y\rangle\rangle \sqsubseteq w$ and (c) $\langle u,\langle Z, Y\rangle\rangle \sqsubseteq\langle\dot{\mathrm{S}} z, \dot{\mathrm{S}} y\rangle$. We prove that that (17) holds in each of these three cases.

Case (a): By $\mathrm{T}_{2}$, we have $u=w, Z=\dot{\mathrm{S}} z$ and $Y=\dot{\mathrm{S}} y$. By (14), we have $z \in N$. By (12), we have $y \in N$. Moreover, by (12), we also have $u=w=\left\langle w^{\prime},\langle z, y\rangle\right\rangle$. Thus there exist $v$ and $Y^{\prime}, Z^{\prime} \in N$ such that

$$
Z=\dot{\mathrm{S}} Z^{\prime} \wedge Y=\dot{\mathrm{S}} Y^{\prime} \wedge u=\left\langle v,\left\langle Z^{\prime}, Y^{\prime}\right\rangle\right\rangle
$$

If $y=\dot{0}$, we must have $\langle v,\langle z, y\rangle\rangle=w=\langle\perp,\langle x, \dot{0}\rangle\rangle$ since $\phi_{A}(x, y, w)$ holds by our assumptions (12). By $\mathrm{T}_{2}$, this implies $z=x$ and $v=\perp$. This proves that (17) holds in Case (a).

Case (b): By our assumptions (12), we have $\phi_{A}(x, y, w)$, and thus we also have $\theta_{A}(u, w, Y, Z) \equiv$

$$
\begin{aligned}
&\langle u,\langle Z, Y\rangle\rangle \sqsubseteq w \wedge Y \neq \dot{0} \rightarrow \\
& \exists v \exists Y^{\prime} Z^{\prime} \in N\left[Z=\dot{\mathrm{S}} Z^{\prime} \wedge Y=\dot{\mathrm{S}} Y^{\prime} \wedge u=\left\langle v,\left\langle Z^{\prime}, Y^{\prime}\right\rangle\right\rangle\right) \wedge \\
&\left.\left(Y^{\prime}=\dot{0} \rightarrow\left(Z^{\prime}=x \wedge v=\perp\right)\right)\right] .
\end{aligned}
$$


We are dealing with a case where the antecedent of (18) holds, and thus (17) holds.

Case (c): This case is not possible. By $\mathrm{T}_{4}$, this case splits into the subcases: (a') $\langle u,\langle Z, Y\rangle\rangle=\langle\dot{\mathrm{S}} z, \dot{\mathrm{S}} y\rangle,\left(\mathrm{b}^{\prime}\right)\langle u,\langle Z, Y\rangle\rangle \sqsubseteq \dot{\mathrm{S}} z$ and $\left(\mathrm{c}^{\prime}\right)\langle u,\langle Z, Y\rangle\rangle \sqsubseteq \dot{\mathrm{S}} y$. We prove that each of these subcases contradicts our axioms. Case (a'): Recall that $\dot{\mathrm{S}} y$ is shorthand for $\langle y, \perp\rangle$. Thus, by $\mathrm{T}_{2}$, we have $Y=\perp$. This contradicts the assumption (12) that $Y \in N$. Case (b'): By Lemma 4 (iii), we have $\langle u,\langle Z, Y\rangle\rangle=\perp \vee N(\langle u,\langle Z, Y\rangle\rangle)$. Now, $\langle u,\langle Z, Y\rangle\rangle=\perp$ contradicts $\mathrm{T}_{1}$. Furthermore, $N(\langle u,\langle Z, Y\rangle\rangle)$ implies that there is $z_{0}$ such that $\langle u,\langle Z, Y\rangle\rangle=\left\langle z_{0}, \perp\right\rangle$. By $\mathrm{T}_{2}$, we have $\langle Z, Y\rangle=\perp$. This contradicts $\mathrm{T}_{1}$. Case (c') is similar to Case (b'). This proves that (17) holds, and thus we conclude that the lemma holds.

\section{Lemma 8.}

$$
\begin{aligned}
& \mathrm{T}^{+} \vdash \forall x y \in N \forall w\left[\phi_{A}(x, \dot{\mathrm{S}} y, w)\right. \rightarrow \\
&\left.\exists u \in N \exists w^{\prime}\left[w=\left\langle w^{\prime},\langle u, \dot{\mathrm{S}} y\rangle\right\rangle \wedge \phi_{A}\left(x, y, w^{\prime}\right)\right]\right] .
\end{aligned}
$$

Proof. Let $x, y \in N$ and assume $\phi_{A}(x, \dot{\mathrm{S}} y, w)$. Thus, we have $w^{\prime}$ and $z \in N$ such that

$$
w=\left\langle w^{\prime},\langle z, \dot{\mathrm{S}} y\rangle\right\rangle \quad \text { and } \quad \forall u \forall Y, Z \in N\left[\theta_{A}(u, w, Y, Z)\right]
$$

Use the assumptions (19) to prove that $\phi_{A}\left(x, y, w^{\prime}\right) \equiv$

$$
\begin{aligned}
\left(y=\dot{0} \rightarrow w^{\prime}=\langle\perp,\langle x, \dot{0}\rangle\rangle\right) \wedge \exists w^{\prime \prime} \exists z \in & N\left[w^{\prime}=\left\langle w^{\prime \prime},\langle z, y\rangle\right\rangle\right] \wedge \\
& \forall u \forall Y, Z \in N\left[\theta_{A}\left(u, w^{\prime}, Y, Z\right)\right]
\end{aligned}
$$

holds. We omit the details.

Lemma 9. The translations of $\mathrm{A}, \mathrm{G}_{4}$ and $\mathrm{G}_{5}$ are theorems of $\mathrm{T}^{+}$.

Proof. The translation of the axiom A is

$$
\forall x, y, z_{1}, z_{2} \in N\left[\Psi_{A}\left(x, y, z_{1}\right) \wedge \Psi_{A}\left(x, y, z_{2}\right) \rightarrow z_{1}=z_{2}\right] .
$$

Assume $\Psi_{A}\left(x, y, z_{1}\right)$ and $\Psi_{A}\left(x, y, z_{2}\right)$. Then it follows straightforwardly from the definition of $\Psi_{A}$ and $\mathrm{T}_{2}$ that $z_{1}=z_{2}$. Hence the translation is a theorem of $\mathrm{T}^{+}$.

The translation of $\mathrm{G}_{4}$ is $\forall x \in N\left[\Psi_{A}(x, \dot{0}, x)\right]$, that is

$$
\begin{aligned}
& \forall x \in N \exists w\left[\phi_{A}(x, \dot{0}, w) \wedge \exists w^{\prime}\left[w=\left\langle w^{\prime},\langle x, \dot{0}\rangle\right\rangle\right] \wedge\right. \\
&\left.\forall u\left[\phi_{A}(x, \dot{0}, u) \rightarrow u=w\right]\right] .
\end{aligned}
$$

We have

$$
\mathrm{T}^{+} \vdash \phi_{A}(x, \dot{0},\langle\perp,\langle x, \dot{0}\rangle\rangle) \text { and } \mathrm{T}^{+} \vdash \forall u\left[\phi_{A}(x, \dot{0}, u) \rightarrow u=\langle\perp,\langle x, \dot{0}\rangle\rangle\right.
$$

by Lemma 6 , and it easy to see that the translation of $\mathrm{G}_{4}$ is a theorem of $\mathrm{T}^{+}$. 
The translation of $\mathrm{G}_{5}$ is

$$
\forall x, y, u \in N\left[\exists z \in N\left[\Psi_{A}(x, y, z) \wedge u=\dot{\mathrm{S}} z\right] \rightarrow \Psi_{A}(x, \dot{\mathrm{S}} y, u)\right]
$$

In order to prove that (21) can be deduced from the axioms of $\mathrm{T}^{+}$, we assume $\Psi_{A}(x, y, z) \wedge u=\dot{\mathrm{S}} z$. Then we need to prove $\Psi_{A}(x, \dot{\mathrm{S}} y, \dot{\mathrm{S}} z) \equiv$

$$
\begin{aligned}
& \exists w\left[\phi_{A}(x, \dot{\mathrm{S}} y, w) \wedge \exists w^{\prime}\left[w=\left\langle w^{\prime},\langle\dot{\mathrm{S}} z, \dot{\mathrm{S}} y\rangle\right\rangle\right] \wedge\right. \\
&\left.\forall u\left[\phi_{A}(x, \dot{\mathrm{S}} y, u) \rightarrow u=w\right]\right] .
\end{aligned}
$$

By our assumption $\Psi_{A}(x, y, z)$ there is a unique $w_{1}$ such that $\phi_{A}\left(x, y, w_{1}\right)$ and $w_{1}=\left\langle w_{0},\langle z, y\rangle\right\rangle$ for some $w_{0}$. By Lemma 7 , we have $\phi_{A}\left(x, \dot{\mathrm{S}} y,\left\langle w_{1},\langle\dot{\mathrm{S}} z, \dot{\mathrm{S}} y\rangle\right\rangle\right)$. Thus, we have $w_{2}$ such that $\phi_{A}\left(x, \dot{\mathrm{S}} y, w_{2}\right)$ and $w_{2}=\left\langle w_{1},\langle\dot{\mathrm{S}} z, \dot{\mathrm{S}} y\rangle\right\rangle$. It is easy to see that (22) holds if $w_{2}$ is unique. Thus we are left to prove the uniqueness of $w_{2}$, more precisely, we need to prove that

$$
\forall W_{2}\left[\phi_{A}\left(x, \dot{\mathrm{S}} y, W_{2}\right) \rightarrow W_{2}=w_{2}\right]
$$

In order to prove (23), we assume $\phi_{A}\left(x, \dot{\mathrm{S}} y, W_{2}\right)$ (we will prove $W_{2}=w_{2}=$ $\left.\left\langle w_{1},\langle\dot{\mathrm{S}} z, \dot{\mathrm{S}} y\rangle\right\rangle\right)$. By our assumption $\phi_{A}\left(x, \dot{\mathrm{S}} y, W_{2}\right)$ and Lemma 8 , we have $u_{0} \in$ $N$ and $W_{1}$ such that $W_{2}=\left\langle W_{1},\left\langle u_{0}, \dot{\mathrm{S}} y\right\rangle\right\rangle$ and $\phi_{A}\left(x, y, W_{1}\right)$. We have argued that there is a unique $w_{1}=\left\langle w_{0},\langle z, y\rangle\right\rangle$ such that $\phi_{A}\left(x, y, w_{1}\right)$ holds. By this uniqueness, we have $W_{1}=w_{1}=\left\langle w_{0},\langle z, y\rangle\right\rangle$. So far we have proved

$$
w_{2}=\langle\overbrace{\left\langle w_{0},\langle z, y\rangle\right\rangle}^{w_{1}},\langle\dot{\mathrm{S}} z, \dot{\mathrm{S}} y\rangle\rangle \text { and } W_{2}=\langle\overbrace{\left\langle w_{0},\langle z, y\rangle\right\rangle}^{W_{1}},\left\langle u_{0}, \dot{\mathrm{S}} y\right\rangle\rangle
$$

and then we are left to prove that $u_{0}=\dot{\mathrm{S}} z$. By our assumption $\phi_{A}\left(x, \dot{\mathrm{S}} y, W_{2}\right)$, we have $v$ and $Z^{\prime}, Y^{\prime} \in N$ such that $u_{0}=\dot{\mathrm{S}} Z^{\prime}, \dot{\mathrm{S}} y=\dot{\mathrm{S}} Y^{\prime}$ and $W_{1}=\left\langle v,\left\langle Z^{\prime}, Y^{\prime}\right\rangle\right\rangle$. Thus, $\left\langle v,\left\langle Z^{\prime}, Y^{\prime}\right\rangle\right\rangle=\left\langle w_{0},\langle z, y\rangle\right\rangle$. By $\mathrm{T}_{2}$, we have $z=Z^{\prime}$, and thus, $u_{0}=\dot{\mathrm{S}} Z^{\prime}=$ $\dot{\mathrm{S}} z$. This proves that $(23)$ holds.

We will now give the translation $\Psi_{M}$ of $M$. Let $\phi_{M}(x, y, w) \equiv$

$$
\begin{aligned}
(y=\dot{0} \rightarrow w=\langle\perp,\langle\dot{0}, \dot{0}\rangle\rangle) \wedge \exists w^{\prime} \exists z \in N\left[w=\left\langle w^{\prime},\langle z, y\rangle\right\rangle\right] \wedge \\
\forall u \forall Y, Z \in N \theta_{M}(u, w, Y, Z)
\end{aligned}
$$

where $\theta_{M}(u, w, Y, Z) \equiv$

$$
\begin{aligned}
\langle u,\langle Z, Y\rangle\rangle \sqsubseteq w \wedge Y \neq \dot{0} \rightarrow \exists v \exists Y^{\prime}, Z^{\prime} \in N\left[\Psi_{A}\left(Z^{\prime}, x, Z\right) \wedge\right. \\
\left.Y=\dot{\mathrm{S}} Y^{\prime} \wedge u=\left\langle v,\left\langle Z^{\prime}, Y^{\prime}\right\rangle\right\rangle \wedge\left(Y^{\prime}=\dot{0} \rightarrow Z^{\prime}=\dot{0} \wedge v=\perp\right)\right] .
\end{aligned}
$$

We let $\Psi_{M}(x, y, z) \equiv$

$$
\exists w\left[\phi_{M}(x, y, w) \wedge \exists w^{\prime}\left[w=\left\langle w^{\prime},\langle z, y\rangle\right\rangle \wedge \forall u\left[\phi_{M}(x, y, u) \rightarrow u=w\right]\right] .\right.
$$


The translations of $M, G_{6}$ and $G_{7}$ are

$$
\begin{aligned}
& \mathrm{M} \forall x, y, z_{1}, z_{2} \in N\left[\Psi_{M}\left(x, y, z_{1}\right) \wedge \Psi_{M}\left(x, y, z_{2}\right) \rightarrow z_{1}=z_{2}\right] \\
& \mathrm{G}_{6} \forall x \in N[M(x, \dot{0}, \dot{0})] \\
& \mathrm{G}_{7} \forall x, y, u \in N\left[\exists z \in N\left[\Psi_{M}(x, y, z) \wedge \Psi_{A}(z, x, u)\right] \rightarrow \Psi_{M}(x, \dot{\mathrm{S}} y, u)\right] .
\end{aligned}
$$

The proof of the next lemma follows the lines of the proof of Lemma 9. We omit the details.

Lemma 10. The translations of $\mathrm{M}, \mathrm{G}_{6}$ and $\mathrm{G}_{7}$ are theorems of $\mathrm{T}^{+}$.

Theorem 11. $\mathrm{Q}$ is interpretable in $\mathrm{T}$.

Proof. It is proved in Svejdar [8] that $\mathrm{Q}$ is interpretable in $\mathrm{Q}^{-}$. It follows from the lemmas above that $\mathrm{Q}^{-}$is interpretable in $\mathrm{T}^{+}$which again is interpretable in T. Hence the theorem holds.

\section{References}

1. Cheng, Y.: Finding the limit of incompleteness I. arXiv:1902.06658v2

2. Damnjanovic, Z.: Mutual interpretability of Robinson arithmetic and adjunctive set theory. Bull. Symb. Logic 23, 381-404 (2017)

3. Grzegorczyk, A., Zdanowski, K.: Undecidability and concatenation. In: Ehrenfeucht, A., et al. (eds.) Andrzej Mostowski and Foundational Studies, pp. 72-91. IOS, Amsterdam (2008)

4. Higuchi, K., Horihata, Y.: Weak theories of concatenation and minimal essentially undecidable theories. Arch. Math. Logic 53, 835-853 (2014). https://doi.org/10. 1007/s00153-014-0391-x

5. Jerabek, E.: Recursive functions and existentially closed structures. J. Math. Logic (2019). https://doi.org/10.1142/S0219061320500026

6. Jones, J., Shepherdson, J.: Variants of Robinson's essentially undecidable theory $R$. Archiv für mathematische Logik und Grundlagenforschung 23, 61-64 (1983)

7. Kristiansen, L., Murwanashyaka, J.: First-order concatenation theory with bounded quantifiers. Arch. Math. Logic (accepted)

8. Svejdar, V.: An interpretation of Robinson arithmetic in its Grzegorczyk's weaker variant. Fundamenta Informaticae 81, 347-354 (2007)

9. Tarski, A., Mostowski, A., Robinson, R.M.: Undecidable Theories. North-Holland, Amsterdam (1953)

10. Visser, A.: Growing commas. A study of sequentiality and concatenation. Notre Dame J. Formal Logic 50, 61-85 (2009)

11. Visser, A.: Why the theory $R$ is special. In: Tennant, N. (ed.) Foundational Adventures. Essays in Honour of Harvey Friedman, pp. 7-23. College Publications, UK (2014) 